\title{
ON THE CONCEPT OF $\varphi$-ENTROPY
}

\author{
MEHDI RAHIMI
}

\begin{abstract}
In this paper, the concept of $\varphi$-entropy is defined and some of the its properties are proved. It is a type of generalized entropy with generalized properties. It is invariant under topological conjugacy and satisfies a generalized version of Jaccob's Theorem. Finally, we will extract the Kolmogorov entropy as a special case, by setting $\varphi$ to be the identity function.
\end{abstract}

MSC 2010. 37A35.

Key words. Entropy, $\varphi$-entropy, invariant.

\section{REFERENCES}

[1] R.L. Adler, A.G. Konheim and M.H. McAndrew, Topological entropy, Trans. Amer. Math. Soc., 114 (1965), 309-319.

[2] S. Arimoto, Information-theoretic considerations on estimation problems, Information and Control, 19 (1971), 181-190.

[3] M. Belis and S. Guiasu, A quantitative-qualitative measure of information in cybernetic systems, IEEE Trans. Inform. Theory, 14 (1968), 593-594.

[4] R. Bowen, Invariant measures for Markov maps of the interval, Comm. Math. Phys., 69 (1976), 1-17.

[5] L. Breiman, The individual theorem of information theory, The Annals of Mathematical Statistics, 28 (1957), 809-811; 31 (1960), 809-810 (errata).

[6] M. Brin and A. Katok, On local entropy, in Geometric dynamics, Proceedings of the International Symposium held at the Instituto de Matématica Pura e Aplicada Rio de Janeiro, Brasil, July-August 1981, Lecture Notes in Mathematics, Springer-Verlag, Berlin, 1007 (1983), 30-38.

[7] E.I. Dinaburg, The relation between toological entropy and metric entropy, Soviet Math. Dokl., 11 (1970), 13-16.

[8] C. Ferreri, Hypoentropy and related heterogeneity, divergency and information measures, Statistica, 40 (1980), 155-168.

[9] J. Havrda and F. Charvát, Quantification method of classification processes. Concept of structural a-entropy, Kybernetika, 3 (1967), 30-35.

[10] J.N. Kapur, Generalized entropy of order $\alpha$ and type $\beta$, Mathematics Seminar, Delhi, 4 (1967), 78-94.

[11] J.N. Kapur, Some new non-additive measures of entropy, Boll. U.M.I., 7 (1988), 253266.

The author would like to thank the referee(s) for their comprehensive and useful comments, which helped the improvement of this work to the present form.

DOI: $10.24193 /$ mathcluj.2018.1.07 
[12] A.N. Kolmogorov, New metric invariant of transitive dynamical systems and endomorphisms of Lebesgue spaces, Doklady of Russian Academy of Sciences, 119 (1958), 861864 .

[13] B.H. Lavenda, A new perspective on thermodynamics, Springer, New York, 2010.

[14] B. McMillan, The basic theorems of information theory, The Annals of Mathematical Statistics, 24 (1953), 196-219.

[15] Y. Pesin, Characteristic Lyapunov exponents and smooth ergodic theory, Russian Math. Surveys, 32 (1977), 54-114.

[16] R. Phelps, Lectures on Choquet's Theorem, Van Nostrand, Princeton, 1966.

[17] M. Rahimi and A. Riazi, Entropy operator for continuous dynamical systems of finite topological entropy, Bull. Iranian Math. Soc., 38 (2012), 883-892.

[18] M. Rahimi and A. Riazi, Entropy functional for continuous systems of finite entropy, Acta Math. Sci. Ser. B Engl. Ed., 32 (2012), 775-782.

[19] P.N. Rathie, On a generalized entropy and a coding theorem, J. Appl. Probab., 7 (1970), 124-133.

[20] A. Rényi, On the measures of entropy and information, in Proc. 4th Berkely Symp. Math. Statist. and Probl., University of California Press, 1 (1961), 547-461.

[21] D. Ruelle, An inequality for the entropy of differential maps, Bull. Braz. Math. Soc., 9 (1978), 83-87.

[22] A.P. Sant'Anna and I.J. Taneja, Trigonometric entropies, Jensen difference divergence measures and error bounds, Inform. Sci., 35 (1985), 145-155.

[23] C. Shannon, A mathematical theory of communication, The Bell System Technical Journal, 27 (1948), 379-423, 623-656.

[24] B.D. Sharma and D.P. Mittal, New nonadditive measures of inaccuracy, Journal of Mathematical Sciences, 10 (1975), 122-133.

[25] B.D. Sharma and I.J. Taneja, Entropy of type $(\alpha, \beta)$ and other generalized additive measures in information theory, Metrika, 22 (1975), 205-215.

[26] B.D. Sharma and I.J. Taneja, Three generalized additive measures of entropy, Elec. Inform. Kybern., 13 (1977), 419-433.

[27] Y.G. Sinai, On the notion of entropy of a dynamical system, Doklady of Russian Academy of Sciences, 124 (1959), 768-771.

[28] R.S. Varma, Generalizations of Reyni's Entropy of Order $\alpha$, Journal of Mathematical Sciences, 1 (1966), 34-48.

[29] P. Walters, An introduction to ergodic theory, Springer-Verlag, New York, 1982.

Received September 4, 2017

Accepted November 4, 2017

\author{
University of Qom \\ Faculty of Science \\ Department of Mathematics \\ Qom, Iran \\ E-mail: m10.rahimi@gmail.com
}

